\title{
Avian Diversity in Mizoram University Campus, Aizawl, Mizoram
}

\author{
Lalawmawia Sailo $^{1}$, G.S. Solanki ${ }^{2 *}$ and C. Lalhruaizela ${ }^{3}$ \\ ${ }^{1,2}$ Department of Zoology, Mizoram University \\ ${ }^{3}$ Department of Journalism \& Mass Communication, Mizoram University \\ E-mail: *gssolanki02@yahoo.co.in
}

\begin{abstract}
We conducted an avian survey to assess the avian diversity of Mizoram University campus, Aizawl for a period of 24 months. During the study a total of 3,555 no of individuals of 189 species of birds belonging to 43 families were recorded. The family Muscicapidae represented the highest species composition with 28 species followed by family Timaliidae and Cuculidae with 19 and 13 species respectively. Avian profile of university campus, 1419(74.5\%) are known to be resident of MZU campus and its adjacent area, $31(16.4 \%)$ species are winter visitors, 15 (7.9\%) species are summer visitor, and two species namely, Hooded pitta (Pitta sordid) and Forest wagtail (Dendronanthus indicus) are passer migrants. The variation in avian species recorded in university campus across the seasons is significant $(\mathrm{P}<0.05)$, variation between the seasons was also significant $(\mathrm{P}<0.01)$. University campus exhibited high avian diversity. Shannon Wiener diversity index $\left(\mathrm{H}^{\prime}\right)$ value calculated was 3.286 and species evenness index was 0.62 . The detailed of avian fauna of campus recoded and the need of conservation actions are discussed in length.
\end{abstract}

\section{INTRODUCTION}

Mizoram falls within the northeast bio-geographical zone and is a part of Indo-Mynmar biodiversity hotspot; encompasses rich biodiversity. Several factors and variability within habitat such as topographical, climatic, and forest category have contributed to avian species diversity and richness. Wilderness species in Mizoram has been reported to have a very wide taxonomic range, with respect to the enormous diversity of ecosystem and geographical conditions. However, in the last few decades, human activities and infrastructure developmental projects are the primary factors liable for reduction of biodiversity and also resulted in reduced carrying capacity of the environment (Gaston et al., 2003). Human induced disturbances are causing subtle to major landscape changes. Rapid deforestation of tropical forest and large scale human disturbances has increased concern about their effect on vegetation structure and composition, and animal communities in general (Schutle and Niemi, 1998).

Birds are more sensitive to such changes and are considered good predictors of habitat quality, as they relate to changes in their associated habitat in numerous ways
(Shankar Raman et al. 1998, Chettri et al. 2001, Shankar Raman, 2011) because they respond to habitat structure (MacArthur and MacArthur, 1961) and represent several trophic groups or guilds (Steele et al. 1984). Mizoram has a great variety of wild flora and fauna due to its location within Indo-Myanmar Hotspot region. Bird community plays an important role in forest ecosystem. Although population studies can be used for monitoring the long term change in the ecosystem (Weins, 1989), knowledge of the diversity of bird in a particular area is important for determining the health of the ecosystem. The requirement for bird's habitat is food, water, space and cover (US Fish and Wildlife Service (2002). So, the study of the avian community of a particular habitat can be a determinant of the health of that habitat. Mizoram University campus is lush green campus with high biological diversity. Since campus is relatively new and vibrant due to various infrastructural and anthropogenic activities. Landscape and natural forest transformation is undergoing rapidly that is affecting natural fauna. A study was undertaken to develop a profile and assess avian community on the campus that will give a baseline data for further studies on monitoring and evaluation of avian fauna when university campus will grow further and witness more such activities. 


\section{MATERIALS AND METHOD}

\section{STUdY AREA}

Study was carried out inside the Mizoram University (MZU) campus, $15 \mathrm{Km}$ away from Aizawl town. Mizoram University campus encompasses roughly 980 acres of land area and geographically located between $23^{\circ} 73^{\prime} 94^{\prime \prime} \mathrm{N}$ and $92^{\circ} 66^{\prime} 51^{\prime \prime}$ E elevation ranges from $300 \mathrm{~m}$ to $880 \mathrm{~m}$ above mean sea level. Number of steams flows through the campus namely Setlak lui, Rultawi lui, Hradawng lui, Lalmangkhawng lui, Lungsumazau lui, Kel lui and Chengkawl lui flows through campus and joins the main Tlawng river (Zothanpui, 2019). The vegetation type is mainly tropical wet evergreen to semi evergreen including a protected forest and a small biodiversity park. The area was covered with lush green vegetation with mostly evergreen trees of 384 species of vascular plants which belongs to 290 genera and 107 families (Lalchhuanawma, 2008) interspersed with tall grass.

The bird survey was conducted inside the University campus and its adjacent forest by walking the forested path. Opportunistic sampling was also considered to strengthen the species composition. The survey path were walked mostly in the morning (0500-0830hrs) and evening (1400 -1700hrs) for 24 months during 2017and 2018. Frequency of observing birds was maintained one day per week and four days in a month. For each survey, DSLR Camera (Nikon D5100 and Canon 760D) was used for photography and binocular (Nikon Aculon15x50) was used for observation and identification of birds, For identification of birds, colored plates of Grimmett et al. (2013) were used. A book on Popular Birds of Mizoram (Lalthanzara and Kasambe, 2015) was referred for local name of birds. Variation in avian diversity across the seasons was tested by Analysis of Variance (ANOVA) and comparison of diversity between the seasons was tested by student's ' $t$ ' test using SPSS ver. 17. Shannon Weaver's diversity and Simpson evenness index was calculated as per Mugurran (1985).

\section{OBSERVATIONS AND DISCUSSION}

Mizoram lies at the Indo-Myanmar Biodiversity hotspot, a biodiversity rich area, however the works on avian community is very scanty. Lepage (2018) recorded 652 species of bird from this hilly state of Mizoram including 26 globally threatened species. Choudhury (2008) listed
479 species with an additional 140 uncertain species from Mizoram. The Zoological Survey of India (2007) recorded 370 species and 317 species by BNHS-ENVIS.The MZU campus is observed an abode for total of 189 species of birds belonging to 43 families (Table 1). The family Muscicapidae has the highest species composition with 28 species recorded inside the campus followed by family Timaliidae and Cuculidae with 19 and 13 species respectively. The familiesZosteropidae, Fringilidae, Rhipiduridae, Aegithinidae, Artamidae, Pittidae, Eurylaimidae, Coraciidae, Podargidae, Caprimulgidae, Upupidae, Rallidae, and Turnicidae has a single species representative (Fig. 1).

The high species composition of the family Muscicapidae is recorded, it might be due to the higher adaptability of members of the family in areas of various anthropogenic disturbances and tolerant of various threats. Their habits of foraging in the top canopy, open shrub and near human settlements are also believed to increase the species count in the present study. It may be also due to the diverse habitat occupied by various species under the family Muscicapidae which allows the species to thrive well and survive under the dynamic ecosystem in MZU campus with ongoing diverse anthropogenic activities.

A total of 3,555 no of individuals of bird belonging to 189 different species recorded during total observation period indicate high degree of avian diversity that is also reflected in Shannon Wiener diversity index $(\mathrm{H})$ of 3.287. There thirteen families which are represented by single species is another indicator of species diversity. Distribution of species evenness was high with 0.62. This high diversity of bird species in a roughly 980 acres of land was remarkably high. The result of evenness clearly depicts the occurrence of individuals of different species which in turn indicates the suitability of the habitat for a great variety of bird species. 141 species were identified as resident, 31 species were winter visitors (table 2), 15 were summer visitor (table 3), and two species were recorded as passer migrants, Hooded pitta (Pitta sordid) and Forest wagtail (Dendronanthus indicus) (table 4). A photoplate of some birds is also enclosed. Variation of avian species across the seasons was found to be significant $(\mathrm{P}<0.05)$ and species recorded between seasons was compared and also found significant $(\mathrm{P}<0.01)$. The present record of 189 species at MZU campus is more than the record elsewhere in similar habitat. Chakdar et al. (2016) recorded 73 species of birds in the Assam University Campus of Silchar, Assam. Dey et al. (2013) recorded 76 species of birds from 234 acre campus 
area of Maharaja Bir Bikram College, Agartala, Tripura. Mizoram University is a potential habitat for avian diversity in comparison to other such organizations in this region.

In Dampa Tiger Reserve (DTR), 215 species of birds was claimed to be present by the official website of Environment, Forest and Climate Change Department, Government of Mizoram. The renowned Murlen National Park (MNP) is known to be haven for more than 150 species of birds while Lalawmawia and Lalthanzara (2015) recorded 146 species of birds from Lengteng Wildlife Sanctuary. Meanwhile, Vanlalsawmi et al. (2011) also reported 54 species of birds only from DTR in a short study period. The avian species diversity of MZU campus is higher than the Phawngpui National Park (PNP) where 108 species are recorded by Ghose (1999). However inter species and inter generic variations may be higher in Phawngpui nationa Park being a protected area.
Among the recorded avian species, $75 \%$ are resident of MZU campus and its adjacent area and $25 \%$ of birds are visitors (Fig. 2). The grassy patch which interspersed the evergreen forest traversed by the wet and dried streams provide safe haven for these large congregation of diverse avian species. High percentage of resident species clearly depicts the richness of MZU campus in terms of avian diversity make this area very important for conservation and rehabilitation of birds. The authors encounter with some local hunters inside the MZU campus that is distressing for birds, and need immediate intervention of the University authorities. In view of the high number of resident species as well as seasonal visitors, the campus area needs to be well preserved and protected from the onslaught of external and internal threats which are deleterious to the wildlife and their habitat.

Table 1: Check List of Birds Recorded on Mizoram University Campus

\begin{tabular}{|c|c|c|c|}
\hline Family & English Name & Scientific Name & Local Name( Mizo) \\
\hline \multirow[t]{3}{*}{ Phasianidae } & Red Jungle Fowl & Gallus gallus & Ram-ar \\
\hline & Kalij Pheasant & Lophura leucomelanos & Vahrit \\
\hline & Mountain Bamboo Partridge & Bambusicola fytchii & Vahlah \\
\hline Turnicidae & Barred Button Quail & Turnix suscitator & Vahmim \\
\hline \multirow[t]{2}{*}{ Falconidae } & Amur Falcon & Falco amurensis & Sialsir \\
\hline & Common Kestrel & Falco tinnunculus & Mu te \\
\hline \multirow[t]{9}{*}{ Accipitridae } & Crested Serpent Eagle & Spilornis cheela & Muvanlai \\
\hline & Oriental Honey Buzzard & Pernis ptilorynchus & Khuaimu \\
\hline & Crested Goshawk & Accipiter trivirgatus & Muningaldang \\
\hline & Shikra & Accipiter badius & Mute \\
\hline & Japanese Sparrowhawk & Accipiter gularis & Mute \\
\hline & Besra & Accipiter virgatus & Mute \\
\hline & Rufous-bellied Eagle & Lophotriorchis kienerii & Mu-ar la \\
\hline & Common Buzzard & Buteo buteo & Munibuang \\
\hline & Black Baza & Aviceda leuphotes & Mu kelrang \\
\hline
\end{tabular}

Table 1 (Contd.)... 


\section{Avian Diversity in Mizoram University Campus, Aizawl, Mizoram}

...Table 1 (Contd.)

\begin{tabular}{|c|c|c|c|}
\hline Family & English Name & Scientific Name & Local Name( Mizo) \\
\hline Rallidae & Slaty-legged Crake & Rallina eurizonoides & Kang-kang \\
\hline \multirow[t]{8}{*}{ Culumbidae } & Pin-tailed Green Pigeon & Treron apicauda & Huipui \\
\hline & Orange-breasted Green Pigeon & Treron bicinctus & Vahui \\
\hline & Wedge-tailed Green Pigeon & Treron sphenurus & Vahui \\
\hline & Barred Cuckoo Dove & Macropygia unchall & Thumimeisei \\
\hline & Emerald Dove & Chalcophaps indica & Ramparva \\
\hline & Spotted Dove & Stigmatopelia chinensis & Thuro \\
\hline & Oriental Turtle Dove & Streptopelia orientalis & Thumi \\
\hline & Thick-billed Green Pigeon & Treron curvirostra & Huifek \\
\hline \multirow[t]{2}{*}{ Psittacidae } & Red-breasted Parakeet & Psittacula alexandri & Kiteng \\
\hline & Vernal Hanging Parrot & Loriculus vernalis & Run vaki/Vaki te \\
\hline \multirow[t]{6}{*}{ Strigidae } & Himalayan Wood Owl & Strix leptogrammica & Chingpirinu \\
\hline & Collered Owlet & Glaucidium brodiei & Hrangkir \\
\hline & Collared Scops Owl & Otus lettia & Chhimbuk \\
\hline & Oriental Scops Owl & Otus sunia & Chhimbuk \\
\hline & Asian-barred Owlet & Glaucidium cuculoides & Chhimbuk te \\
\hline & Spot-bellied Eagle Owl & Bubo nipalensis & Chhimbukpui \\
\hline \multirow[t]{8}{*}{ Cuculidae } & Greater Coucal & Centropus sinensis & Lalruangasehnawt \\
\hline & Lesser Coucal & Centropus bengalensis & Lalruangasehnawt \\
\hline & Green-billed Malkoha & Rhopodytes tristis & Vazun/va uk \\
\hline & Large Hawk Cuckoo & Hierococcyх sparverioides & Kiltheihrawk \\
\hline & Common Hawk Cuckoo & Hierococcyx varius & Kiltheihrawk \\
\hline & Asian Koel & Eudynamys scolopaceus & Mitthi ar \\
\hline & Jacobin Cuckoo & Clamator jacobinus & \\
\hline & Chestnut-winged cuckoo & Clamator coromandus & \\
\hline
\end{tabular}

Table 1 (Contd.).. 
... Table 1 (Contd.)

\begin{tabular}{|c|c|c|c|}
\hline Family & English Name & Scientific Name & Local Name( Mizo) \\
\hline & Banded Bay Cuckoo & Cacomantis sonneratii & Thangfenpabawp \\
\hline & Asian Emerald Cuckoo & Chrysococcyx maculatus & \\
\hline & Lesser Cuckoo & Cuculus poliocephalus & Thangfenpabawp te zawk \\
\hline & Hodgson's Hawk Cuckoo & Hierococcyx nisicolor & Kiltheihrawk \\
\hline & Violet Cuckoo & Chrysococcyx xanthorynchus & Mawntaipirtliak \\
\hline \multirow[t]{2}{*}{ Meropidae } & Blue-bearded Bee-eater & Nyctyornis athertoni & Tlakawrh \\
\hline & Chestnut-headed Bee-eater & Merops leschenaulti & Fuanhawr \\
\hline Upupidae & Common Hoopoe & Upupa epops & Chhuangtuar/Vaseek \\
\hline \multirow[t]{2}{*}{ Ramphastidae } & Blue-throated Barbet & Megalaima asiatica & Tukloh \\
\hline & Great Barbet & Megalaima virens & Tawllawt \\
\hline \multirow[t]{10}{*}{ Picidae } & Greater Yellownape & Picus flavinucha & Thlohlupar \\
\hline & Lesser Yellownape & Picus chlorolophus & Thlohlupar \\
\hline & White-browed Piculet & Sasia ochraceae & Luangtubeuh \\
\hline & Speckled Piculet & Picumnus innominatus & Luangtubeuh \\
\hline & $\begin{array}{l}\text { Grey-capped Pygmy } \\
\text { Woodpecker }\end{array}$ & Dendrocopos canicapillus & Thlohte \\
\hline & Fulvous-breasted Woodpecker & Dendrocopos macei & Thlohte \\
\hline & Bay Woodpecker & Blythipicus pyrrhotis & Mauthloh \\
\hline & Rufous Woodpecker & Micropternus brachyurus & Fanghmir thloh \\
\hline & Eurasian Wryneck & Jynx torquilla & Valeisei \\
\hline & Grey-headed Woodpecker & Picus canus & Thloh hring \\
\hline \multirow[t]{5}{*}{ Hirundinidae } & Nepal House Martin & Delichon nipalense & Vamurte \\
\hline & Asian Palm Swift & Cypsiurus balasiensis & Vamur \\
\hline & Brown -backed Needletail & Hirundapus giganteus & Murpui \\
\hline & Fork-tailed Swift & Apus pacificus & Murpui \\
\hline & Striated Swallow & Cecropis striolata & Vamur Ngumsen \\
\hline
\end{tabular}

Table 1 (Contd.)... 


\section{Avian Diversity in Mizoram University Campus, Aizawl, Mizoram}

...Table 1 (Contd.)

\begin{tabular}{|c|c|c|c|}
\hline Family & English Name & Scientific Name & Local Name( Mizo) \\
\hline \multirow[t]{4}{*}{ Motacillidae } & Grey Wagtail & Motacilla cinerea & Lailen \\
\hline & White Wagtail & Motacilla alba & Lailen var \\
\hline & Tree Pipit & Anthus triavilis & Chip \\
\hline & Forest Wagtail & Dendronanthus indicus & Sehnungzui/Ngawkar Lailen \\
\hline \multirow[t]{6}{*}{ Campephagidae } & Scarlet Minivet & Pericrocotus speciosus & Bawng \\
\hline & Long-tailed Minivet & Pericrocotus ethologus & Bawng \\
\hline & Ashy Minivet & Pericrocotus divaricatus & Bawngte \\
\hline & Large Woodshrike & Tephrodornis gularis & Thlekbur \\
\hline & Large Cuckooshrike & Coracina macei & Irliak \\
\hline & Black-winged Cuckooshrike & Coracina melaschistos & Changde \\
\hline \multirow[t]{7}{*}{ Pycnonotidae } & Red-vented Bulbul & Pycnonotus cafer & Tlaiberh \\
\hline & Red-whiskered Bulbul & Pycnonotus jacosus & Thlangvaberh/Phaitlaiberh \\
\hline & Black-crested Bulbul & Pycnonotus flaviventris & Tukkhumvilik \\
\hline & White-throated Bulbul & Alphophoixus flaveolus & Dawkek \\
\hline & Ashy Bulbul & Hemixos flavala & Kawlrit \\
\hline & Flavescent Bulbul & Pycnonotus flavescens & Setawt \\
\hline & Black Bulbul & Hypsipetes leucocephalus & Hmuisen/Kesen/Liandorit \\
\hline Caprimulgidae & Grey Nightjar & Caprimulgus jotaka & Valambawk \\
\hline Podargidae & Hodgson's Frogmouth & Batrachostomus hodgsoni & Valambawk/Vabak \\
\hline Coraciidae & Indian Roller & Coracias benghalensis & Vapui \\
\hline Eurylaimidae & Long-tailed Broadbill & Psarisomus dalhousiae & Thizil \\
\hline Pittidae & Hooded Pitta & Pitta sordida & Buarchawm lu uk \\
\hline Artamidae & Ashy Wood swallow & Artamus fuscus & Lengder \\
\hline Aegithinidae & Common iora & Aegithina tiphia & Zairumva \\
\hline Laniidae & Long-tailed Shrike & Lanius scach & Chhemhur \\
\hline Halcyonidae & Brown Shrike & Lanius cristatus & Chhemhur uk \\
\hline
\end{tabular}

Table 1 (Contd.)... 
...Table 1 (Contd.)

\begin{tabular}{|c|c|c|c|}
\hline Family & English Name & Scientific Name & Local Name( Mizo) \\
\hline & Burmese Shrike & Lanius collurioides & Chhemhur sen uk \\
\hline & Grey-backed Shrike & Lanius tephronotus & Chhemhur(vut buak) \\
\hline & White-throated Kingfisher & Halcyon smyrnensis & Kaikuangral \\
\hline & Black-capped Kingfisher & Halcyon pileata & Kaikuangral ludum \\
\hline \multirow[t]{5}{*}{ Dicruridae } & Bronzed Drongo & Dicrurus aeneus & Changkak \\
\hline & Ashy Drongo & Dicrurus leucophaeus & Thlanthla \\
\hline & Lesser Racket-tailed Drongo & Dicrurus remifer & Thlanthla changhlawi \\
\hline & Greater Racket-tailed Drongo & Dicrurus paradiseus & Vakul changhlawi \\
\hline & Hair-crested Drongo & Dicrurus hottentottus & Kulherh \\
\hline \multirow[t]{3}{*}{ Oriolidae } & Slender-billed Oriole & Oriolus tenuirostris & Vamaitai \\
\hline & Black-naped Oriole & Oriolus chinensis & Vamaitai \\
\hline & Maroon Oriole & Oriolus trailii & Changsen \\
\hline Rhipiduridae & White-throated Fantail & Rhipidura albicollis & Changarh \\
\hline \multirow[t]{2}{*}{ Monarchidae } & Black-naped Monarch & Hypothymis azurae & Zumzek/Thangthlengral \\
\hline & Asian Paradise Flycatcher & Terpsiphone paradisi & Thlehhniar \\
\hline \multirow[t]{4}{*}{ Corvidae } & Eastern Jungle Crow & Corvus macrorynchos & Choak \\
\hline & Common Green Magpie & Cissa chinensis & Dawntliang \\
\hline & Rufous Treepie & Dendrocitta vagabunda & Bemkawng \\
\hline & Grey Treepie & Dendrocitta formosae & Bemkawng \\
\hline \multirow[t]{5}{*}{ Cisticolidae } & Common Tailorbird & Orthotomus sutorius & Daikat/hnahkhawr \\
\hline & Plain Prinia & Prinia inornata & Zirziak \\
\hline & Striated Prinia & Prinia crinigera & Changdawt \\
\hline & Black-throated Prinia & prinia atrogularis & Changdawt awr dum \\
\hline & Rufescent Prinia & Prinia rufescens & Zirziak \\
\hline Sylviidae & Yellow-browed Leaf Warbler & Phyloscopus inornatus & $\begin{array}{l}\text { Chivit/Saivate/Chilim/ } \\
\text { Chhawlchhaih }\end{array}$ \\
\hline
\end{tabular}

Table 1 (Contd.)... 


\section{Avian Diversity in Mizoram University Campus, Aizawl, Mizoram}

... Table 1 (Contd.)

\begin{tabular}{|c|c|c|c|}
\hline Family & English Name & Scientific Name & Local Name( Mizo) \\
\hline & Grey-crowned Warbler & Seicercus burkii & $\begin{array}{l}\text { Chivit/Saivate/Chilim/ } \\
\text { Chhawlchhaih }\end{array}$ \\
\hline & Yellow-vented Warbler & Phylloscopus cantator & $\begin{array}{l}\text { Chivit/Saivate/Chilim/ } \\
\text { Chhawlchhaih }\end{array}$ \\
\hline & Thick-billed Warbler & Phragamaticola aedon & Hmunchhe arpuilian \\
\hline & Common Stonechat & Saxicola torquatus & Tep \\
\hline & Grey Bushchat & Saxicola ferreus & Terzik \\
\hline \multirow[t]{19}{*}{ Timaliidae } & Puff-throated Babbler & Pellorneum ruficeps & Valeisawt \\
\hline & Rufous-capped Babbler & Stachyridopsis ruficeps & Vate lusen \\
\hline & Pin-striped Tit Babbler & Macronous gularis & \\
\hline & Chestnut-capped Babbler & Timalia pileata & Vatelusen \\
\hline & Rufous-fronted Babbler & Stachyridopsis rufifrons & Vatelusen \\
\hline & Grey-throated Babbler & Stachyris nigriceps & Vatekawngkan \\
\hline & Spot-breasted Scimitar Babbler & Pomatorhinus erythrocnemis & Ngalvapual awmtial \\
\hline & Coral-billed Scimitar Babbler & Pomatorhinus ferruginosus & Ngalvapual \\
\hline & White-crested Laughingthrush & Garrulax leucolophus & Koro \\
\hline & $\begin{array}{l}\text { Greater-necklaced } \\
\text { Laughingthrush }\end{array}$ & Garrulax pectoralis & Vazar/Zarpuithiawrh \\
\hline & $\begin{array}{l}\text { Lesser-necklaced } \\
\text { Laughingthrush }\end{array}$ & Garrulax monileger & Vazar/Zarfek \\
\hline & Rufous-necked Laughingthrush & Garrulax ruficollis & Vachawm \\
\hline & White-browed Shrike Babbler & Pteruthius flaviscapis & Kawl vasir \\
\hline & Brown-cheeked Fulvetta & Alcippe poioicephala & Mau va \\
\hline & Nepal Fulvetta & Alcippe nipalensis & Ngawkar mitval \\
\hline & Yellow-eyed Babbler & Chrysomma sinense & \\
\hline & Striated Yuhina & Staphida castaniceps & Tehhek \\
\hline & White-bellied Erpornis & Erpornis zantholeuca & Vate lungleng \\
\hline & $\begin{array}{l}\text { Greater Rufous-headed } \\
\text { Parrotbill }\end{array}$ & Psittiparus ruficeps & Vahnanghlai chikhat \\
\hline
\end{tabular}

Table 1 (Contd.)... 
...Table 1 (Contd.)

\begin{tabular}{|c|c|c|c|}
\hline Family & English Name & Scientific Name & Local Name( Mizo) \\
\hline \multirow[t]{7}{*}{ Muscicapidae } & White-capped Redstart & Chaimarrornis leucocephalus & Vachalde \\
\hline & Rufous-gorgeted Flycatcher & Ficedula strophiata & Terzik nghawngsen \\
\hline & Little Pied Flycatcher & & Ter pa \\
\hline & Verditer Flycatcher & Eumyias thalassinus & Vapawl \\
\hline & Taiga Flycatcher & ficedula albicilla & Ter \\
\hline & Blue Whistling Thrush & Myophonus caeruleus & Thangfenpabawp \\
\hline & Orange-headed Thrush & Zoothera citrina & Vakhuang \\
\hline & Scaly Thrush & Zoothera dauma & Ram chippui \\
\hline & Grey-sided Thrush & Turdus faea & Tiau \\
\hline & Blue Rock Thrush & Monticola solitarius & Va-in-ro nghak \\
\hline & Long-billed Thrush & Zoothera monticola & Ramchippui hmuisei \\
\hline & Dark-sided Thrush & Zoothera marginata & Ramchippui \\
\hline & Siberian Rubythroat & Luscinia calliope & Tawktawk awrsen \\
\hline & White-tailed Rubythroat & Luscinia pectoralis & Tawktawk awrsen(a chang var) \\
\hline & Himalayan Bluetail & Tarsiger rufilatus & \\
\hline & Oriental Magpie Robin & Copsychus saularis & Khawmual chinrang \\
\hline & White-rumped Shama & Copsychus malabaricus & Vatelal \\
\hline & Black Redstart & Phoenicurus ochruros & \\
\hline & Blue-fronted Redstart & Phoenicurus frontalis & \\
\hline & Black-backed Forktail & Enicurus immaculatus & Chinrang \\
\hline & Spotted Forktail & Enicuruc maculatus & Chinrang \\
\hline & White-tailed Robin & Myiomela leucura & Pi-tuibur-kei-ve/Pi tuibur dil \\
\hline & Dark-sided Flycatcher & Muscicapa sibirica & Ter \\
\hline & Asian Brown Flycatcher & Muscicapa dauurica & Ter uk \\
\hline
\end{tabular}

Table 1 (Contd.)... 


\section{Avian Diversity in Mizoram University Campus, Aizawl, Mizoram}

... Table 1 (Contd.)

\begin{tabular}{|c|c|c|c|}
\hline Family & English Name & Scientific Name & Local Name( Mizo) \\
\hline & Vivid Niltava & Niltava vivida & Be-ai-ral \\
\hline & Blue-throated Blue Flycatcher & Cyornis rubiculoides & Vadumdeleng \\
\hline & Small Niltava & Niltava macgrigoriae & Be-ai-ral te zawk \\
\hline & Grey-headed Canary Flycatcher & Culicicapa ceylonensis & Mauhmun vate \\
\hline \multirow[t]{8}{*}{ Nectarinidae } & Crimson Sunbird & Aethopyga siparaja & Dawithiama arpa \\
\hline & Mrs Goulds Sunbird & Aethopyga gouldiae & Dawithiama arpa \\
\hline & Ruby-cheeked Sunbird & Chalcoparia singalensis & Dawithiama arpa \\
\hline & Little Spiderhunter & Arachnothera longirostra & $\begin{array}{l}\text { Kireuh te/Tumbu ar/Zetzet/ } \\
\text { Lawizit }\end{array}$ \\
\hline & Streaked Spiderhunter & Arachnothera magna & Kireuh \\
\hline & Plain Flowerpecker & Dicaeum minullum & Tiktik \\
\hline & Yellow-vented Flowerpecker & Dicaeum chrysorrheum & Tiktik awmtial \\
\hline & Scarlet-backed Flowerpecker & Dicaeum cruentatum & Tek tek \\
\hline \multirow[t]{2}{*}{ Chloropseidae } & Orange-bellied Leafbird & Chloropsis hardwickii & Chhawlhring awm eng \\
\hline & Golden-fronted Leafbird & Chloropsis aurifrons & Chhawlhring lu sen \\
\hline Fringilidae & Common Rosefinch & Carpodacus erythrinus & Vasuih \\
\hline \multirow[t]{2}{*}{ Estrildidae } & Little Bunting & Emberiza pusilla & Ram chawngzawng \\
\hline & Crested Bunting & Melophus lathami & Ram chawngzawngpui \\
\hline Zosteropidae & Oriental White-eye & Zosterops palpebrosus & Mitval/Pirh/Uichirh \\
\hline \multirow[t]{2}{*}{ Sittidae } & Chestnut-vented Nuthatch & Sitta nagaensis & Suklet \\
\hline & Velvet-fronted Nuthatch & Sitta frontalis & Suklet \\
\hline \multirow[t]{2}{*}{ Sturnidae } & Chestnut-tailed Starling & Sturnia malabarica & Vamam/Vapaw \\
\hline & Common Hill Myna & Gracula religiosa & Vaiva \\
\hline \multirow[t]{3}{*}{ Passeridae } & Eurasian Tree Sparrow & Passer montanus & Chawngzawng \\
\hline & Scaly-breasted Munia & Lonchura punctulata & Pit awmtial \\
\hline & White-rumped Munia & Lonchura striata & Pit(ngumvar) \\
\hline
\end{tabular}




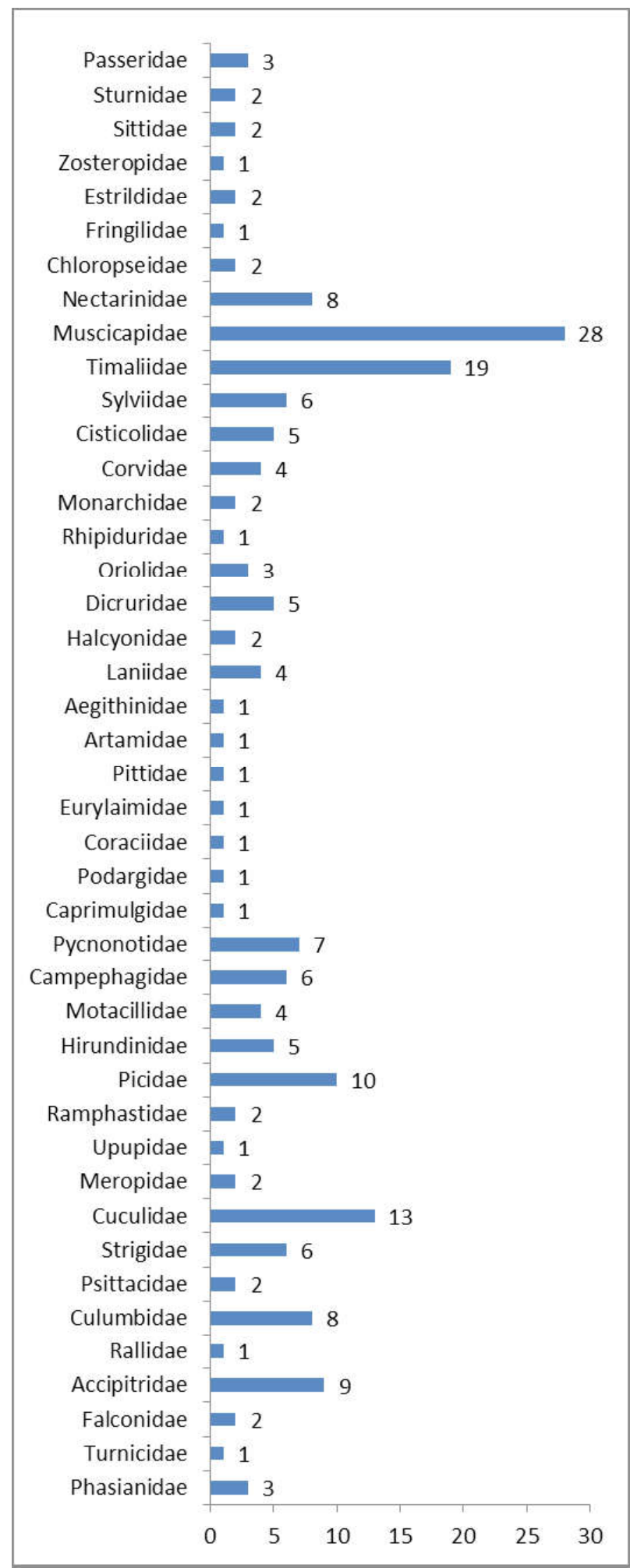

Fig. 1: Summary of Bird Species Recorded 


\section{Avian Diversity in Mizoram University Campus, Aizawl, Mizoram}

Table 2: Birds Recoded during Winter Season (Winter Visitors)

\begin{tabular}{|c|c|c|c|}
\hline Family & English Name & Scientific Name & Mizo Name \\
\hline \multirow[t]{2}{*}{ Falconidae } & Amur Falcon & Falco amurensis & Sialsir \\
\hline & Common Kestrel & Falco tinnunculus & Mu te \\
\hline \multirow[t]{3}{*}{ Accipitridae } & Oriental Honey Buzzard & Pernis ptilorynchus & Khuaimu \\
\hline & Japanese Sparrowhawk & Accipiter gularis & Mute \\
\hline & Common Buzzard & Buteo buteo & Munibuang \\
\hline \multirow[t]{2}{*}{ Psittasidae } & Red-breasted Parakeet & Psittacula alexandri & Kiteng \\
\hline & Vernal Hanging Parrot & Loriculus vernalis & Run vaki/Vaki te \\
\hline Meropidae & Chestnut-headed Bee-eater & Merops leschenaulti & Fuanhawr \\
\hline Upupidae & Common Hoopoe & Upupa ерорs & Chhuangtuar/Vaseek \\
\hline Picidae & Eurasian Wryneck & Jynx torquilla & Valeisei \\
\hline \multirow[t]{2}{*}{ Hirundinidae } & Brown -backed Needletail & Hirundapus giganteus & Murpui \\
\hline & Fork-tailed Swift & Apus pacificus & Murpui \\
\hline \multirow[t]{2}{*}{ Motacilidae } & Grey Wagtail & Motacilla cinerea & Lailen \\
\hline & White Wagtail & Motacilla alba & Lailen var \\
\hline \multirow[t]{4}{*}{ Sylviidae } & $\begin{array}{l}\text { Yellow-browed Leaf } \\
\text { Warbler }\end{array}$ & Phyloscopus inornatus & Chivit/Saivate/Chilim/Chhawlchhaih \\
\hline & Grey-crowned Warbler & Seicercus burkii & Chivit/Saivate/Chilim/Chhawlchhaih \\
\hline & Yellow-vented Warbler & phylloscopus cantator & Chivit/Saivate/Chilim/Chhawlchhaih \\
\hline & Common Stonechat & Saxicola torquatus & Tep \\
\hline \multirow[t]{12}{*}{ Muscicapidae } & White-capped Redstart & $\begin{array}{l}\text { Chaimarrornis } \\
\text { leucocephalus }\end{array}$ & Vachalde \\
\hline & Verditer Flycatcher & Eumyias thalassinus & Vapawl \\
\hline & Taiga Flycatcher & ficedula albicilla & Ter \\
\hline & Grey-sided Thrush & Turdus faea & Tiau \\
\hline & Long-billed Thrush & Zoothera monticola & Ramchippui hmuisei \\
\hline & Dark-sided Thrush & Zoothera marginata & Ramchippui \\
\hline & Siberian Rubythroat & Luscinia calliope & Tawktawk awrsen \\
\hline & White-tailed Rubythroat & Luscinia pectoralis & Tawktawk awrsen(a chang var) \\
\hline & Himalayan Bluetail & Tarsiger rufilatus & \\
\hline & Blue-fronted Redstart & Phoenicurus frontalis & \\
\hline & Dark-sided Flycatcher & Muscicapa sibirica & Ter \\
\hline & Asian Brown Flycatcher & Muscicapa dauurica & Ter uk \\
\hline Fringilidae & Common Rosefinch & Carpodacus erythrinus & Vasuih \\
\hline
\end{tabular}


Table 3: Birds Recoded during Summer Season (Summer Visitors)

\begin{tabular}{|l|l|l|l|}
\hline \multicolumn{1}{|c|}{ Family } & \multicolumn{1}{|c|}{ English Name } & \multicolumn{1}{c|}{ Mizo Name } \\
\hline \multirow{5}{*}{ Cuculidae } & Large Hawk Cuckoo & Hierococcyx sparverioides & Kiltheihrawk \\
\cline { 2 - 4 } & Common Hawk Cuckoo & Hierococcyx varius & Kiltheihrawk \\
\cline { 2 - 4 } & Asian Koel & Eudynamys scolopaceus & Mitthi ar \\
\cline { 2 - 4 } & Jacobin Cuckoo & Clamator jacobinus & \\
\cline { 2 - 4 } & Chestnut-winged cuckoo & Clamator coromandus & Thangfenpabawp \\
\cline { 2 - 4 } & Banded Bay Cuckoo & Cacomantis sonneratii & \\
\cline { 2 - 4 } & Asian Emerald Cuckoo & Chrysococcyx maculatus & Thangfenpabawp te zawk \\
\cline { 2 - 4 } & Lesser Cuckoo & Cuculus poliocephalus & Kiltheihrawk \\
\cline { 2 - 4 } & Hodgson's Hawk Cuckoo & Hierococcyx nisicolor & Mawntaipirtliak \\
\cline { 2 - 4 } & Violet Cuckoo & Chrysococcyx xanthorynchus & Vamurte \\
\hline \multirow{5}{*}{ Hirundinidae } & Nepal House Martin & Delichon nipalense & Be-ai-ral \\
\hline Muscicapidae & Vivid Niltava & Niltava vivida & Vadumdeleng \\
\cline { 2 - 4 } & Blue-throated Blue Flycatcher & Cyornis rubiculoides & Be-ai-ral te zawk \\
\cline { 2 - 4 } & Small Niltava & Niltava macgrigoriae & Vakhuang \\
\cline { 2 - 3 } & Orange-headed Thrush & Zoothera citrina & \\
\hline Total no of families: 3 and species: 15 are summer visitors & & \\
\hline
\end{tabular}

Table 4: Passage Migrant Birds

\begin{tabular}{|l|l|l|l|}
\hline \multicolumn{1}{|c|}{ Family } & \multicolumn{1}{|c|}{ English Name } & \multicolumn{1}{c|}{ Scientific Name } & \multicolumn{1}{c|}{ Mizo Name } \\
\hline Pittidae & Hooded Pitta & Pitta sordida & Buarchawm lu uk \\
\hline Motacillidae & Forest Wagtail & Dendronanthus indicus & Sehnungzui/Ngawkar Lailen \\
\hline
\end{tabular}

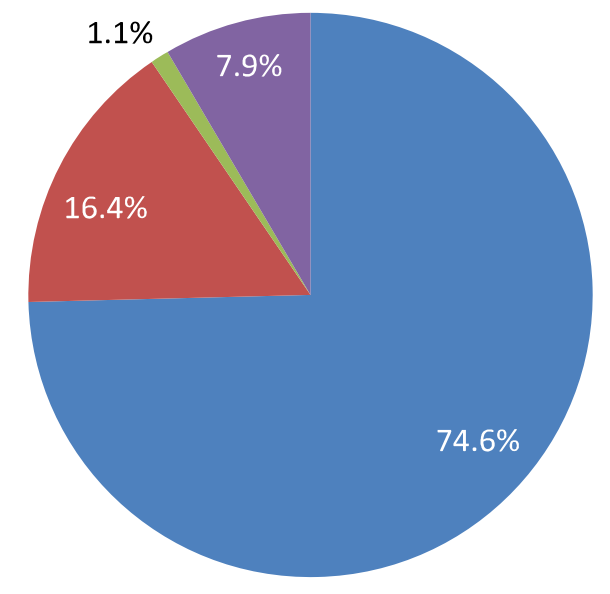

Resident ${ }^{\circledR}$

Winter visitor (W)

Passage migrant (PM)

Summer (S)

Fig. 2: Patterns of Occurrence of Avian Groups 


\section{Avian Diversity in Mizoram University Campus, Aizawl, Mizoram}

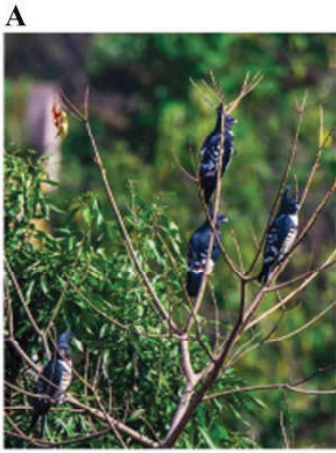

Black Baza (Aviceda leuphotes)

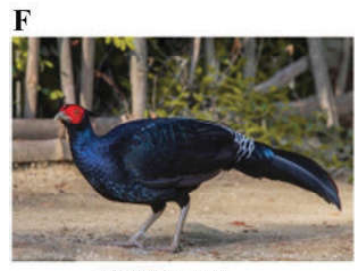

Kalij Pheasant

(Lophura leucomelanos)

J

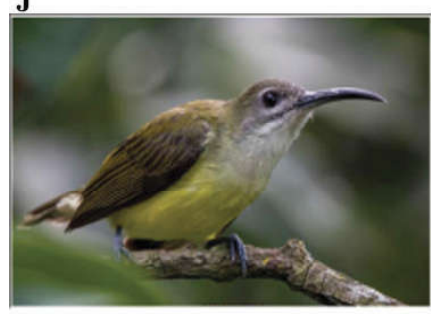

Little Spiderhunter (Arachnothera longirostra)

B

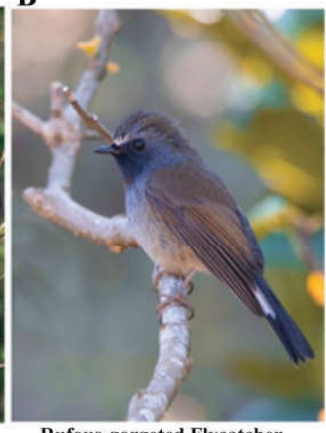

Rufous-gorgeted Flycatcher

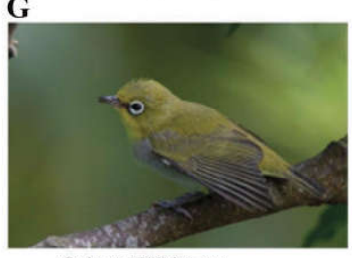

Oriental White-eye (Zosterops palpebrosus)

$\mathbf{K}$

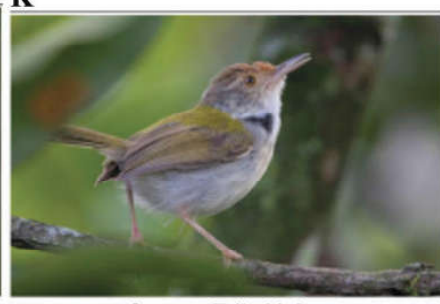

Common Tailorbird (Orthotomus sutorius)
C

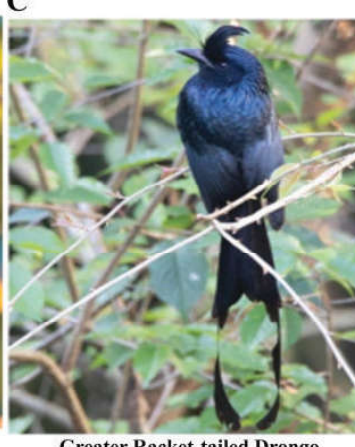

Greater Racket-tailed Drong H
D

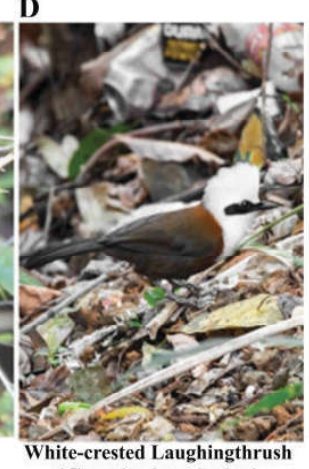

(Garrulax leucolophus)

E
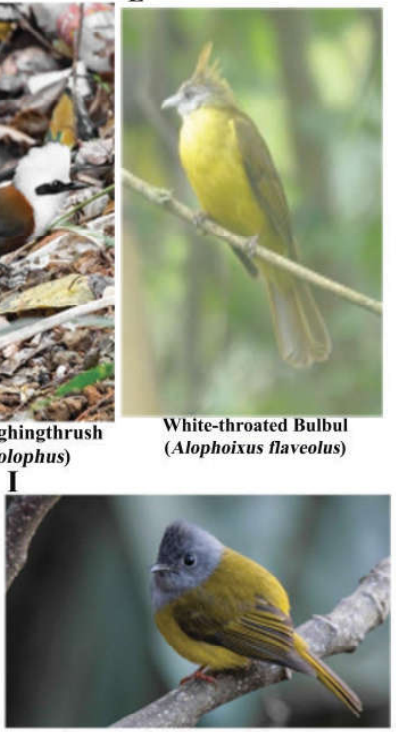

Grey-headed Canary Flycatcher (Culicicapa ceylonensis)

M

L

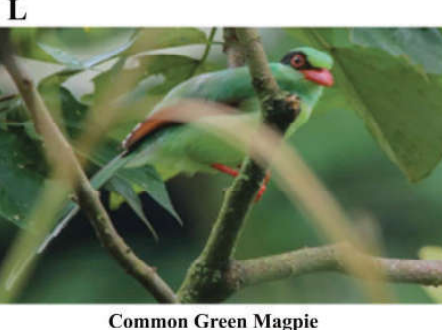

Common Green Magpie

(Cissa chinensis)

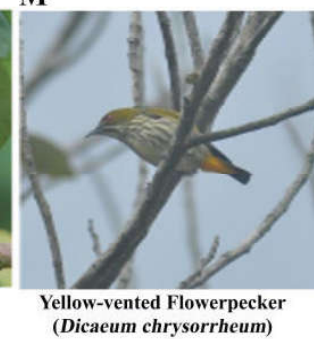

Fig. 3: Photoplate: Some Birds of Mizoram University (MZU) Campus

\section{REFERENCES}

Environment and forest. Birds; 2019 [cited on 27.7.19]. Available from: http://envformizo.in/forest/birds.htm

Chakdar, B., Choudhury, P. and Singha, H. 2016. Avifaunal Diversity in Assam University Campus, Silchar, India. J. of Threat. Taxa 8(1):8369-8378.

Chettri, N., Sharma, E. and Deb, D. C. 2001. Bird community structure along a trekking corridor of Sikkim Himalaya:A conservation Perspective. Biological conservation, 12:150-158.

Choudhury, A. 2008. A Pocket Guide to the Birds of Mizoram. Gibbon Books, Guwahati, Assam and The Rhino Foundation for Nature in NE India, Guwahati, Assam, India, pp 1-122.

Dey, A., Deb, D., Chaudhuri, S. D. and Chaudhuri, P. S. 2013. A Preliminary Study on Avifaunal Species Diversity of Maharaja Bir Bikram College Campus, Tripura, North East India. Int. Multidisc. Res. J., 2013, 3(2):36-43

Envis Centre on Avian Ecology. Statewise list of Birds of India. 2019 [cited on 7.8.2019] Available from: http://www.bnhsenvis. nic.in/Database/Statewise\%.
Gaston, K. J., Blackburn, T. M. and Goldewijk, K. K. 2003. Habitat conversion and global avian biodiversity loss. Proc. R. Soc. Lond. B (2003) 270, 1923-1300.

Ghose, D. 1999. Birds recorded at Blue Mountain (Phawngpui) National Park, Mizoram Between February - May, 1997. Twilight, 1,16-18.

Grimmett, R., Inskipp, C. and Inskipp, T. 2011. Birds of the Indian Subcontinent (Second Edition). Oxford University Press, India. Pages 528.

Lalchhuanawma. 2008. Ecological studies on plant diversity and productivity of herbaceous species in Mizoram Universityu campus at Tanhril, Aizawl, Mizoram (N.E.India). Ph.D. thesis submitted to Mizoram University.

Lalthanzara, H. 2010. Recent status of threatened birds of Mizoram. Sci Vis, 10 (4), 168-169.

Lalthanzara, H., Kasambe, R. 2015. Popular Birds of Mizoram. Scientific Book Centre, Guwahati.Pages124

Lalthanzara, H., Lalramliana, Vanramliana, Lalnunzira, Vanlalsiama, Liana, J.P. 2011. Blyth's Tragopan (Tragopan blythii) in Lengteng Wildlife Sanctuary, Mizoram, India. Sci. Vis., 11(2), 108-112. 
Lalthanzara, H., Ramanujam, S.N., Solanki, G.S. and Sailo, L. 2013. Survey on distribution of pheasants (Galliformes) in Mizoram, India. Sci. Vis., 13(2), 90-95.

Lalthanzara, H., Sailo, L., Solanki, G.S. and Ramanujam, S.N. 2014. Galliformes and Their Conservation Issues in Mizoram, India. Cibtech J Zool, 3(1), 42-48.

Lalthanzara, H., Sailo, L., Solanki, G.S., Ramanujam, S,N, Lallianthanga, R.K. and Lalbiakmawia. 2014. Grey Peacock Pheasant (Polyplectron bicalcaratum) as probable candidate for Ecological Indicator in Tropical Montane Forest of Mizoram, Northeast India. In Lalnuntluanga et al. Eds. Issues and Trends of Wildlife Conservation in Northeast India. pp. 233-239.

Lalthanzara, H., Vanramliana and Lalramliana. 2011. Pheasants of Mizoram (India): Present status of diversity and distribution. Sci. Vis., 11(4), 218-223.

Lepage, D. 2018. Checklists of Birds of Mizoram. Avibase, the world bird database. Retrieved from http://avibase.bsc-eoc. org/checklist.jsp?region=INnemz\&list=howardmoore on 08.07.2019.

McArthur, R.H. and McArthur, J.W. 1961. On bird species diversity. Ecology 42:594-599.

Manakadan, R. and Pittie, A. 2001. Standardised common and scientific names of the birds of the Indian subcontinent. Buceros 6(1): 1-37.

Magurran, A. E. 1985. Measuring Biological diversity. Black Well Publishing company, UK.

Schulte, L.A. and Niemi, G. J. 1998.Bird communities of early successional burned and logged forest. J. Wildlife Mgmt.62:1418-1429.
Shankar Raman, T.R., Rawat, G.S. and Johnsingh, A. J. T. 1998. Recovery of tropical rainforest avifauna in relation to vegetation succession following shifting cultivation in Mizoram, north-east India. J. Appl. Ecol., 35, 214-231.

Shankar Raman, T.R. 2001. Effect of slash-and -burn shifting cultivation on rainfgorested birds in Mizoram, Northeast, India. Conser. Biol.,15:685-698.

Steele, B.B., Bayn, R.L.Jr. and Grant, C.V. 1984. Environmental Monitoring using population of birds and small mammals: Analysis of sampling efforts. Biol. Conser., 30:157-172.

US Fish and Wildlife Service. The four essential Elements of Habitat. 2002. [cited on 12.8.2019]. Available from: https:// digitalmedia.fws.gov/digital/collection/document/ id/1426/

Vanlalsawmi, R., Solanki, G.S. and Zakhuma. 2011. Birds of Dampa Tiger Reserve in Mizoram, India. Proceedings of Advances in Environmental Chemistry, pp. 242-244.

Wiens, J.A. 1989. The ecology of the bird communities Vol.1.Foundation and pattern Cambridge University press.

Zothanpuii, J.H. 2019. Faunal diversity and distribution within Mizoram Univesity campus using camera trap technique. M.Sc. dissertation submitted for part fulfillment of degree of Master of Science in Zoology. Mizoram University, Aizawl. Pages 40.

Zoological Survey of India. 2007. Fauna of Mizoram. State Fauna Series 14, pp 1-691. 6. 